\title{
On the Trigonometric Correction of One Powerful Formula
}

\author{
Jiří Stávek ${ }^{1}$ \\ ${ }^{1}$ Bazovského 1228, 16300 Prague, Czech Republic \\ Correspondence: Jiří Stávek, Bazovského 1228, 16300 Prague, Czech Republic. E-mail: stavek.jiri@seznam.cz
}

Received: December 30, 2014 Accepted: January 16, 2015 Online Published: January 28, 2015

doi:10.5539/apr.v7n2p14

URL: http://dx.doi.org/10.5539/apr.v7n2p14

\begin{abstract}
An attempt is presented for the description of the magnitude of Newton's gravitational force in the experiments with a horizontal torsion balance. There were developed many experimental arrangements in order to find experimentally the value of big $\mathrm{G}$ - the Newtonian gravitational constant - after the Michell-Cavendish experiment in 1798. The geometrical configurations of test and source masses play a very important role in these experiments. The old trigonometric function "sagitta" used by Johannes Kepler and Isaac Newton was newly employed as the trigonometric tool for the determination of the magnitude of Newton's gravitational force between the source mass and the test mass. Based on the known experimental configurations with the horizontal torsion balance we have found that the "true" Michell-Cavendish configuration is not dependent on the space orientation. This "sagitta" function can be experimentally tested in the Karagioz-Izmailov configuration and the Karagioz-Izmailov-Gillies-Gershteyn configuration with the technology available at begin of our century. A proposal for the decomposition of the big $\mathrm{G}$ was presented. This concept could not be experimentally tested before the discoveries of the dipole in the cosmic microwave background radiation and the Pioneer anomaly.
\end{abstract}

Keywords: Principia, trigonometric function "sagitta", direction of gravitational force, magnitude of gravitational force, horizontal torsion balance, Michell-Cavendish configuration, Karagioz-Izmailov configuration, Karagioz-Izmailov-Gillies-Gershteyn configuration, gravitational waves around the moving source mass, decomposition of big $\mathrm{G}$.

\section{Introduction}

Gravitational interaction, described by the Newton's law (1687) of gravitation $\mathrm{F}=\mathrm{Gm}_{1} \mathrm{~m}_{2} / \mathrm{d}^{2}$ where $\mathrm{F}$ represents force of attraction between two particles having masses $m_{1}$ and $m_{2}$ and $d$ is the distance between these particles, continues to attract experimental and theoretical researchers. While the absolute values of other fundamental constants are known with high accuracy, the accuracy of experimental determination of $\mathrm{G}$ is below that of other fundamental constants. There are two main actual topics in this feld of the experimental physics. From one side the development of the experimental techniques steadily continues to design more precise instruments in order to come closer to the absolute value of G. From the other side the experimental research is focused on the elimination and description of numerous factors bringing their influence on the data distribution of obtained $G$ values in different top laboratories. A determination of $G$ is conceptually easy: measure the force two known masses arranged in a known geometry and compare the result with the Newton's law of gravitation. But the experimental realization of these measurements requires the greatest attention to all parameters: many of them are already known but some of them are still hidden. Big G measurements are full of elegance, complexity, subtlety, and beauty. Experimentalists try to answer the permanent question: can we find some hidden parameters behind these experiments and to find mathematical tools for their quantitative description and prediction?

The actual state of the art of the experimental determination of $\mathrm{G}$ was recently summarized in the special issue of the Philosophical Transactions A (2014): "Theo Murphy Meeting Issue 'The Newtonian constant of gravitation, a constant too difficult to measure?" T.J. Quinn and C.C. Speake concluded that main focus should be given to the explanation of the uncertainty of the value G. Contributions presented at this Meeting surveyed in details the actual experimental situation of the determination of $\mathrm{G}$ and proposed new guidelines for the near future research. B. M. Wood discussed the present situation of gravitational constant experimental results. See all 13 contributions focused on the big $\mathrm{G}$ in this special issue.

The history of the experimental determination of $\mathrm{G}$ is very rich while hundreds of researchers contributed to this development. It is valuable to re-read historical and recent reviews on this topic and to re-visit some original 
manuscripts written by highly skillful researchers. Several older reviews: Baily (1843), Jolly (1881), Poyting (1894), Boys (1895), Eötvös (1896), Richarz and Krigar-Menzel (1898) and the modern review of Ducheyne (2011) covering the years 1798-1898. Many recent reviews can guide us in this research field: Sagitov (1970), Gillies (1987), Michaelis et al. (1995), Gillies (1997), Schwartz (1998), Speake and Quinn (1999), Chen and Cook (1999), Schumacher (1999), Luo and Hu (2000), Kleinevoß (2002), Schlamminger (2002), Speake (2005), Milyukov et al. (2008), Mohr, Taylor and Newell (2008), Milyukov and S. H. Fan (2012), Panjwani (2012).

One aim of this contribution is to present a proposal for a geometric arrangement of the experiments with a horizontal torsion balance to precisely control the directions of the gravitational forces $\mathbf{F}_{1}$ and $\mathbf{F}_{2}$ - the directions of attracting forces between two source masses and two test masses. The second aim of this contribution is to present a possible trigonometric correction for the magnitudes of the gravitational forces $\left|\mathbf{F}_{\mathbf{1}}\right|$ and $\left|\mathbf{F}_{\mathbf{2}}\right|$ - the magnitudes of attracting forces of two source masses on two test masses. Finally, a working formula for the possible decomposition of big $\mathrm{G}$ is presented.

\section{Directions of the Attracting Forces $F_{1}$ and $F_{2}$}

The geometrical arrangement of these experiments plays a very important role. Two test masses are placed on ends of a horizontal torsion balance. This idea was proposed by John Michell and firstly experimentally demonstrated by Henry Cavendish. The positions of two source masses in the space around the test masses have to be precisely defined. During the past two hundred years numerous experimental arrangements were realized in order to study the influence of the spacial positions of the source masses around the test masses while their center of gravity should be identified with the center of the horizontal torsion balance. During last thirty years several new experimental configurations were realized. The most stable values of $\mathrm{G}$ were found for configurations with four source masses and four test masses suspended by a horizontal torsion balance.

There are two methods of measuring the gravitational constant: static and dynamic. The static method was realized by Michell-Cavendish. The dynamic method was developed in several stages by Ferdinand Reich (1852), Karl Braun (1897) and Rolánd Eötvös (1922).

There were proposed several alternatives to the horizontal torsion balance during the $19^{\text {th }}$ century. E.g., Phillip J.G. von Jolly (1881) performed these experiments with a common balance. Johannes Wilsing (1887) used a brass vertical pendulum.

In 1964 Jesse Wakefield Beams proposed a very important innovation for the determination of G. It is a classical Michell-Cavendish horizontal torsion balance but the whole system is placed on virtually frictionless rotary table. In this arrangement we can investigate possible space orientation influences of the attracting forces between the test masses and source masses. The first measured results for this arrangement published Rose et al. (1969). The rotary table experiments were further improved and developed by Gundlach and Merkowitz (2000) and Chao Xue et al. (2014).

\subsection{Gravity Centers of Individual Test Masses and Source Masses Lay on the Same Horizontal Circle}

This is a classical geometrical arrangement proposed by Michell-Cavendish. Two test masses have been placed on a horizontal torsion balance in such a way that their common center of gravity is in the middle of the torsion balance. Two source masses are so positioned that their individual centers of gravity lay on the same horizontal circle and their common center of gravity is identified with the center of the torsion balance. The whole system can be placed on the rotary table and we get the Michell-Cavendish-Beams arrangement.

\subsection{Gravity Centers of Two (or Four) Test Masses Lay on the Inner Horizontal Circle, Gravity Centers of Two (or Four) Source Masses Lay on the Outer Circle and a Combined Configuration}

Two test masses have been placed on a horizontal torsion balance in such a way that their common center of gravity is in the middle of the torsion balance. Horizontal rotation of these test masses forms an inner circle. Two source masses are so positioned that their individual centers of gravity lay on the outer horizontal circle in the same height as the inner circle. The common center of gravity of these source masses is identified with the center of the torsion balance. The whole system can be placed on the rotary table and we get the Heyl-Beams-Rose arrangement Paul R. Heyl (1930) made very important contributions to this kind of experimental arrangement. This horizontal torsion balance system was further developed by Luther and Towler (1982), Quinn and Speake with their coworkers (2001, 2013) (Quinn-Speake configuration), Armstrong and Fitzgerald (2003), Hu and Luo (2004) (Hu-Luo configuration), Luo et al. (2009). 


\subsection{Gravity Centers of Two Test Masses Lay on the Horizontal Circle, Gravity Centers of Source Masses Rotate on a Tilted Circle or Four Source Masses Rotate on Two Tilted Circles}

Two test masses have been placed on a horizontal torsion balance in such a way that their common center of gravity is in the middle of the torsion balance. Rotation of these test masses forms a horizontal circle. Two source masses are placed on a tilted circle above and below the horizontal circle. The common gravity center of two source masses is identified with the gravity center of the torsion balance. We can find precursors for this experimental arrangement in works of Loránd Eötvös (1890), Charles Vernon Boys (1895) and Josef Zahradníček (1933). Therefore, we propose to term this geometrical arrangement as the Eötvös -Boys-Zahradníček configuration. This configuration was recently modified by Gundlach and Merkowitz (2000) - in the Gundlach-Merkowitz configuration four source masses have been used.

\subsection{Gravity Centers of Two Test Masses and Two Source Masses (or One Source Mass) Lay on One Horizontal Line}

Two test masses have been placed on a horizontal torsion balance in such a way that their common center of gravity is in the middle of the torsion balance. Centers of gravity of two source masses (or one source mass) lay on the same horizontal line. This experimental configuration was experimental realized by Karagioz and Izmailov (1996), Karagioz, Izmailov and Gillies (1999) and Gershteyn et al. (2002). See the recent data on the web site of Karagioz and Izmailov (2012). These source masses could be moved individually or together along the horizontal axis. We can term this experimental configuration as Karagioz-Izmailov configuration (with two source masses acting along the horizontal line) and Karagioz-Izmailov-Gillies-Gershteyn configuration (with one source mass acting along the horizontal line). There was a critical discussion to the results found for this configuration published by Unnikrishnan and Gillies (2002).

\subsection{Michell-Cavendish Gravity Probe}

The rotation of two source masses around the two test masses in the 3D space can be described by the mathematical concepts of Claudius Ptolemy, Nicolaus Copernicus, Johannes Kepler and Isaac Newton. Albert Einstein (1915) proposed models for the space with higher dimensions. In the 3D space with the Michell-Cavendish configuration two epicycles (from Greek "circle on circle") are not fictions but can be experimentally realized and their epicycle motions can be observed. Rotation of two test masses $\mathrm{M}_{\mathrm{T}}$ in the horizontal plane forms the deferent circle. Rotation of two source masses $\mathrm{M}_{\mathrm{S}}$ around their test masses forms two epicycles in the 3D space. The gravity center of the whole system is identified with the center of the horizontal torsion balance. This proposed geometrical arrangement of the Michell-Cavendish configuration mounted on a rotary table enables us to study if there might exist a hidden parameter coming from the space orientation of this experiment. See Figure 1.

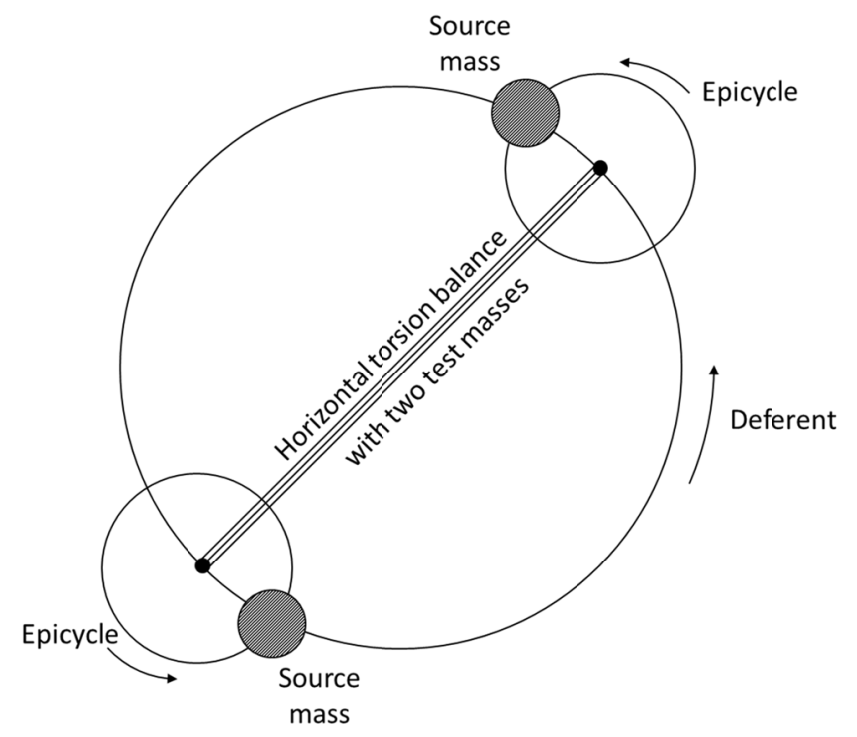

Figure 1. Schema of the rotation of two source masses $M_{S}$ around two test masses $M_{T}$ in the $3 D$ space termed as the Michell-Cavendish configuration 


\section{Trigonometric Tools in the Circle With Diameter $D=1$}

Galileo gave to us his advice "...the letters are triangles, circles and other geometrical figures ..." Galileo's manuscript No. 72 „Notes on Motion“ can be used as a source for the graphical interpretation of the well-known trigonometric functions and to employ them newly for the description of the properties of the gravitational field around moving source masses. Johannes Kepler and Issac Newton used in their works the trigonometric function called "sagitta". Detailed discussions of the trigonometric function "sagitta" in Kepler's and Newton's works can be found in books of D.T. Whiteside, Hoskin and Prag (1974), F. de Gandt (1995), J. B. Brackenridge (1996) and I. B. Cohen (1999).

In order to become more familiar with the trigonometric tool "sagitta" it is valuable to re-read Von Braunmühl (1900), Datta and Singh (1983), Matvieskaya (1990), Maor (1998), Van Brummelen (2009, 2013) and some applications in Stávek (2013).

There are depicted trigonometric functions for the circle with diameter $\mathrm{D}=1$ and the central angle $\varepsilon$ in Figure 2 .

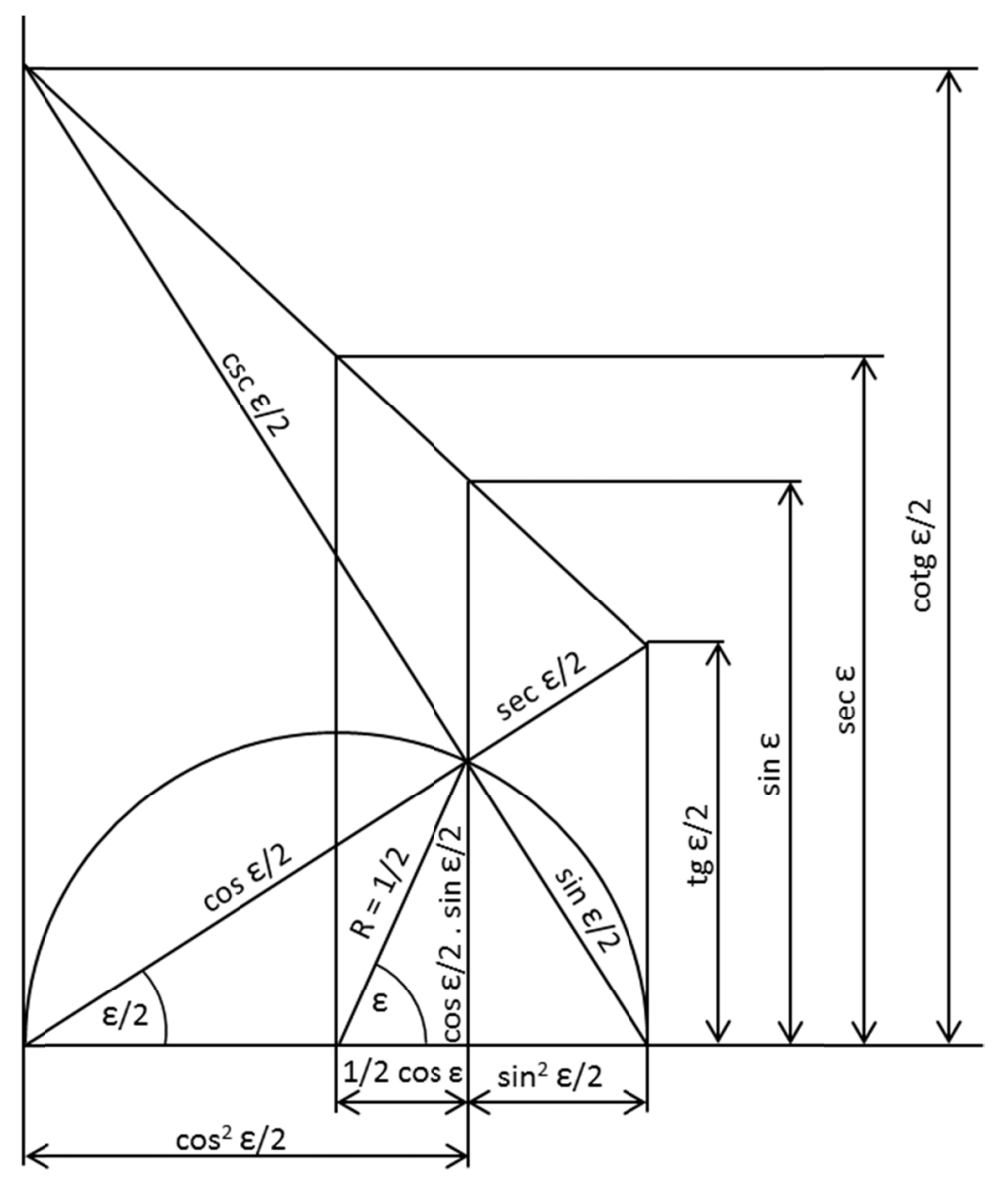

Figure 2. Trigonometric functions in the circle with diameter $\mathrm{D}=1$ and the central angle $\varepsilon$

We can schematically describe the well-known relativistic formulae around the circle with diameter $\mathrm{D}=1$ and the central angle $\varepsilon$ if we will define $1 / 2 \cos (\varepsilon)=1 / 2 * u / c$ in Figure 3 . 


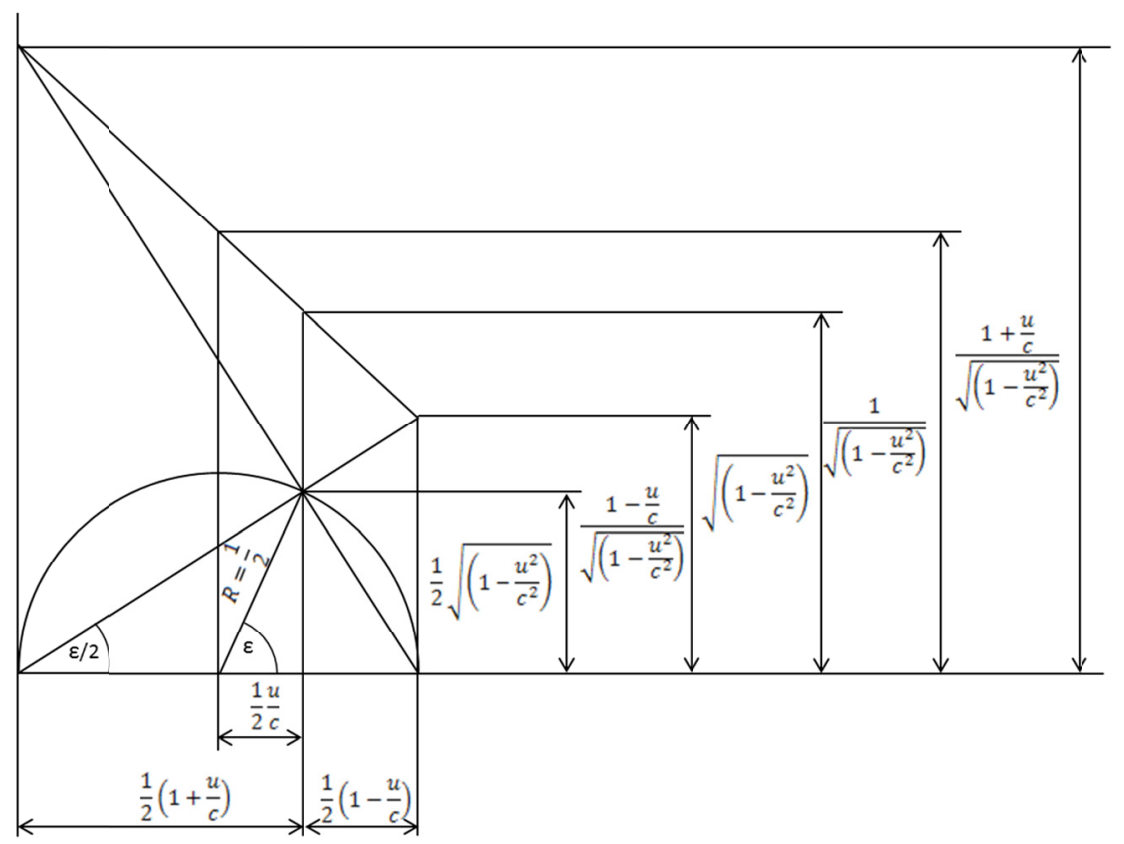

Figure 3. Relativistic formulae around the circle with diameter $\mathrm{D}=1$, the central angle $\varepsilon$ and the defined trigonometric function $1 / 2 \cos (\varepsilon)=1 / 2 * u / c$

Table 1. Trigonometric relations in the circle with diameter $\mathrm{D}=1$ for the defined trigonometric fuction of the central angle: $1 / 2 \cos (\varepsilon)=1 / 2 * \mathrm{u} / \mathrm{c}$

\begin{tabular}{|c|c|c|}
\hline $1 / 2 \cos \varepsilon$ & $1 / 2 \cos \varepsilon$ & $1 / 2 \mathrm{u} / \mathrm{c}$ \\
\hline $1 / 2 \sin \varepsilon$ & $\sin \varepsilon / 2 \cos \varepsilon / 2$ & $\frac{1}{2} \sqrt{1-\frac{u^{2}}{c^{2}}}$ \\
\hline $\operatorname{tg} \varepsilon / 2$ & $\frac{\sin (\varepsilon / 2) \cos (\varepsilon / 2)}{\cos ^{2}(\varepsilon / 2)}$ & $\frac{1-\frac{u}{c}}{\sqrt{1-\frac{u^{2}}{c^{2}}}}$ \\
\hline $\sin \varepsilon$ & $2 \sin \varepsilon / 2 \cos \varepsilon / 2$ & $\sqrt{1-\frac{u^{2}}{c^{2}}}$ \\
\hline $\csc \varepsilon$ & $\frac{1}{\sin \varepsilon}$ & $\frac{1}{\sqrt{1-\frac{u^{2}}{c^{2}}}}$ \\
\hline $\operatorname{cotg} \varepsilon / 2$ & $\frac{\sin (\varepsilon / 2) \cos (\varepsilon / 2)}{\sin ^{2}(\varepsilon / 2)}$ & $\frac{1+\frac{u}{c}}{\sqrt{1-\frac{u^{2}}{c^{2}}}}$ \\
\hline $\csc \varepsilon / 2$ & $\frac{1}{\sin \mathcal{E} / 2}$ & $\sqrt{\frac{2}{1-\frac{u}{c}}}$ \\
\hline $\sec \varepsilon / 2$ & $\frac{1}{\cos \varepsilon / 2}$ & $\sqrt{\frac{2}{1+\frac{u}{c}}}$ \\
\hline
\end{tabular}


We can extract several new valuable relations from Figure 3. The line connecting the values of cotg $(\varepsilon / 2)$ and $\operatorname{tg}$ $(\varepsilon / 2)$ could be described as:

$$
y=\frac{1+\frac{u}{c}-2 \frac{u}{c} \cos ^{2} \frac{\varepsilon}{2}}{\sqrt{1-\frac{u^{2}}{c^{2}}}}
$$

Astrophysicists found from the CMBR dipole anisotropy that the Solar system has been moving in the rest frame of the cosmic microwave background radiation $(\mathrm{CMBR})$ with the speed $\mathrm{u}_{\mathrm{Y}}=369 \pm 0.9 \mathrm{kms}^{-1}$ in the direction $1=$ $263.99^{\circ} \pm 0.14^{\circ}$ and $\mathrm{b}=48.26^{\circ} \pm 0.03^{\circ}$. The direction is towards the constellation Crater close to the boundary with the constellation Leo. The constellation Crater is termed in the Greece mythology as the Cup of the god Apollo. See the web site with Planck mission data (2013). For the timeline of the research of the CMB radiation see the web site of the Wikipedia (2014).

In our concept we will assume that the big $G$ is composed from several parts and one of these parts is the gravitational wavelength $\lambda$ that could be influenced by the source mass motion in the rest frame of the cosmic microwave radiation. In gravitational experiments we should observe the following dependence:

$$
G=\frac{1+\frac{u_{Y}}{c}-2 \frac{u_{Y}}{c} \cos ^{2} \frac{\varepsilon}{2}}{\sqrt{1-\frac{u_{Y}^{2}}{c^{2}}}} G_{0}
$$

The source mass $M_{S}$ has been moving toward to the constellation of the Crater with the speed $u_{Y}$ and the gravitational wave radiating from the source mass $\mathrm{M}_{\mathrm{S}}$ in different directions should be modified by the source mass motion in the CMBR rest frame and can be described by mathematical tools of Christian Doppler (1842), Wilhelm Matzka (1850) and Woldemar Voigt (1887). If this proposed concept agrees with the real situation we should observe in our experiments the gravitational effects depicted by Figure 4.

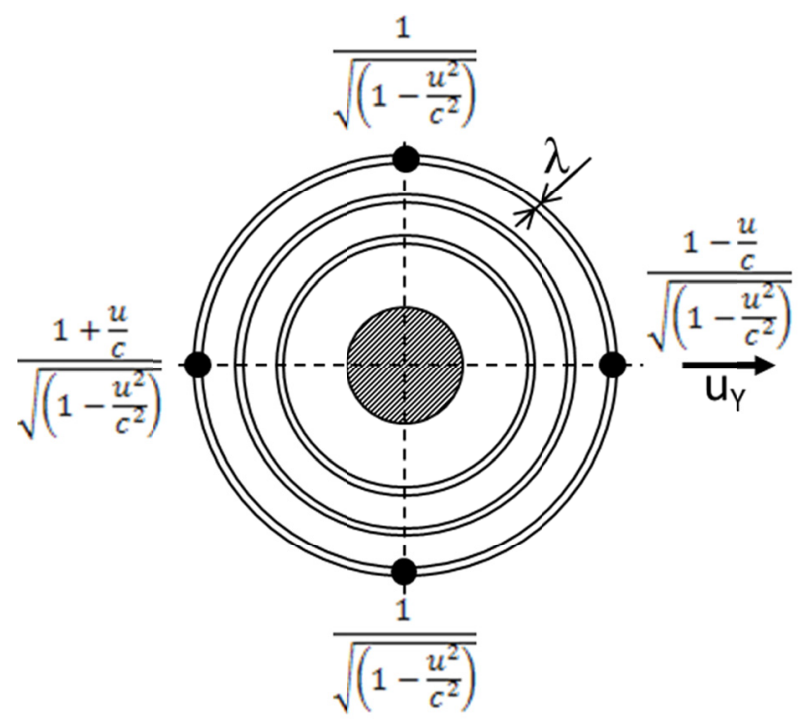

Figure 4. Source mass $\mathrm{M}_{\mathrm{S}}$ radiating gravitational wave in the $\mathrm{CMBR}$ rest

\section{Influence of the Gravity Probe Configuration on the Observed Result}

The geometrical arrangement of these gravitational experiments plays a very important role and can dramatically influence the observed data. We can manipulate with the geometrical configurations of two test masses placed on ends of a horizontal torsion balance and two source masses acting on those test masses. 


\subsection{The "true" Michell-Cavendish Configuration - A Null Experiment}

In this classical geometrical arrangement proposed by Michell and realized by Cavendish two test masses have been placed on a horizontal torsion balance while two source masses have been acting perpendicularly on the torsion balance. The direction of the gravitational field originated from the source masses has to be perpendicular to the torsion balance and not caused by a circular motion of source masses on the deferent circle. Figure 5 depicts a top view of such geometrical arrangement.

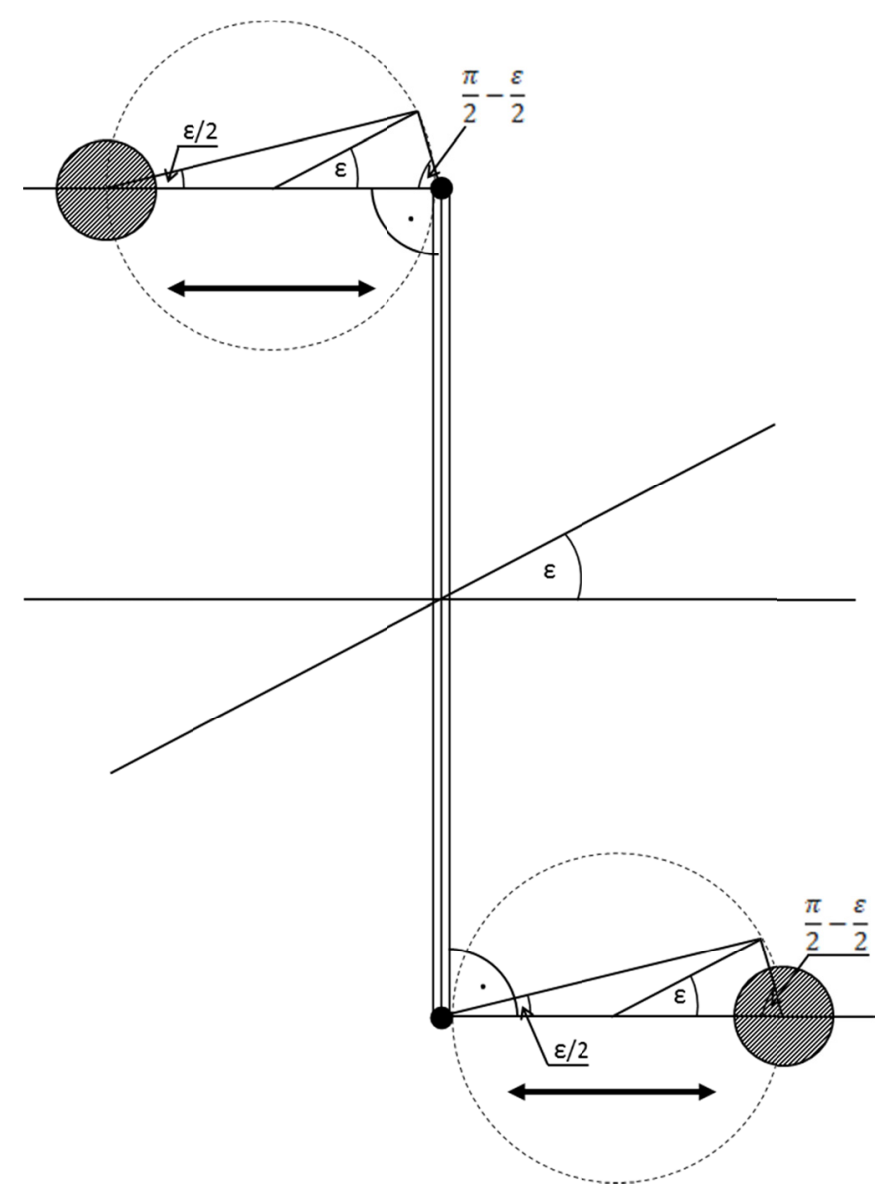

Figure 5. Geometrical configuration of the "true" Michell-Cavendish configuration - a null experiment

For the description of those gravitational forces we will use the trigonometric function "Sagitta" $\sin ^{2}(\varepsilon / 2)$ and $\sin ^{2}(\pi / 2-\varepsilon / 2)$ where $\varepsilon$ is the angle of the horizontal torsion balance towards the constellation Crater. This equation reveals that the "true" Michell-Cavendish configuration is not dependent on the space orientation of this gravitational experiment.

$$
|F|=\frac{1}{2} \frac{1+\frac{u_{Y}}{c}-2 \frac{u_{Y}}{c} \sin ^{2}\left(\frac{\pi}{2}-\frac{\varepsilon}{2}\right)+1+\frac{u_{Y}}{c}-2 \frac{u_{Y}}{c} \sin ^{2} \frac{\varepsilon}{2}}{\sqrt{1-\frac{u_{Y}^{2}}{c^{2}}}} F_{0}=\frac{F_{0}}{\sqrt{1-\frac{u_{Y}^{2}}{c^{2}}}}
$$

\subsection{Karagioz-Izmailov Configuration With Two Source Masses}

In this geometrical arrangement proposed and realized by Karagioz and Izmailov two test masses have been placed on a horizontal torsion balance while two source masses have been acting on the torsion balance to both ends in the direction of the same horizontal line. The direction of the gravitational field originated from the source masses has to lay on the same horizontal line identical with the torsion balance. Figure 6 depicts a top view of this Karagioz-Izmailov arrangement. 


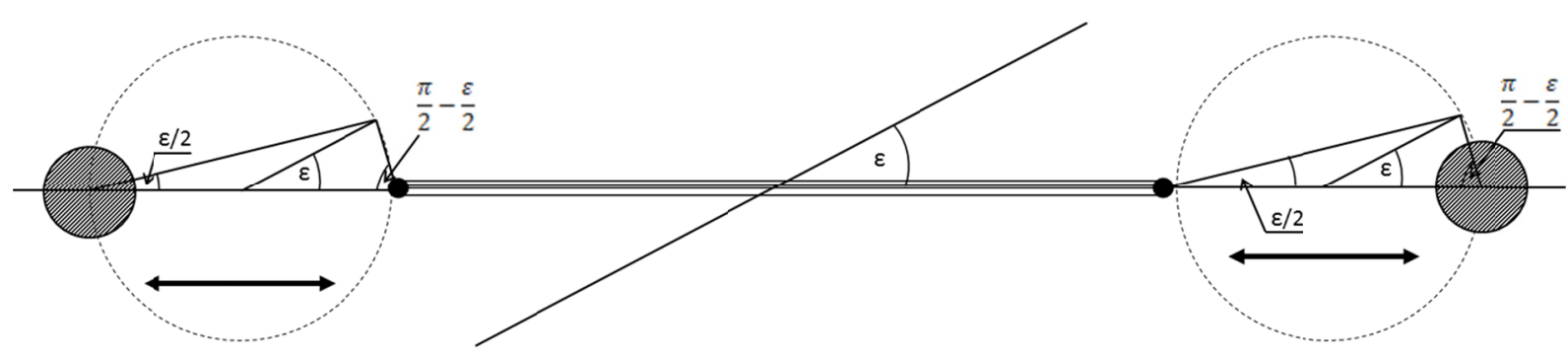

Figure 6. Geometrical configuration of the Karagioz-Izmailov configuration with two source masses

For the description of those gravitational forces we will use the trigonometric function "Sagitta" $\sin ^{2}(\varepsilon / 2)$ and $\sin ^{2}(\pi / 2-\varepsilon / 2)$ where $\varepsilon$ is the angle of the horizontal torsion balance towards the constellation Crater. This equation reveals that the Karagioz-Izmailov configuration is dependent on the space orientation towards the constellation Crater:

$$
\Delta|F|=\frac{1+\frac{u_{Y}}{c}-2 \frac{u_{Y}}{c} \sin ^{2}\left(\frac{\varepsilon}{2}\right)-1-\frac{u_{Y}}{c}+2 \frac{u_{Y}}{c} \sin ^{2}\left(\frac{\pi}{2}-\frac{\varepsilon}{2}\right)}{\sqrt{1-\frac{u_{Y}^{2}}{c^{2}}}}\left|F_{0}\right|=\frac{2 \frac{u_{Y}}{c} \cos (\varepsilon)}{\sqrt{1-\frac{u_{Y}^{2}}{c^{2}}}}\left|F_{0}\right|
$$

\subsection{Karagioz-Izmailov-Gillies-Gershteyn Configuration With One Source Mass}

In this geometrical arrangement proposed by Karagioz, Izmailov and Gillies and realized by Gershteyn et al. two test masses have been placed on a horizontal torsion balance while one source mass has been acting on the torsion balance to one end in the same horizontal line. The direction of the gravitational field originated from the source mass has to be along the same horizontal line of the torsion balance. Figure 7 shows a top view of this Karagioz-Izmailov-Gillies-Gershteyn configuration.

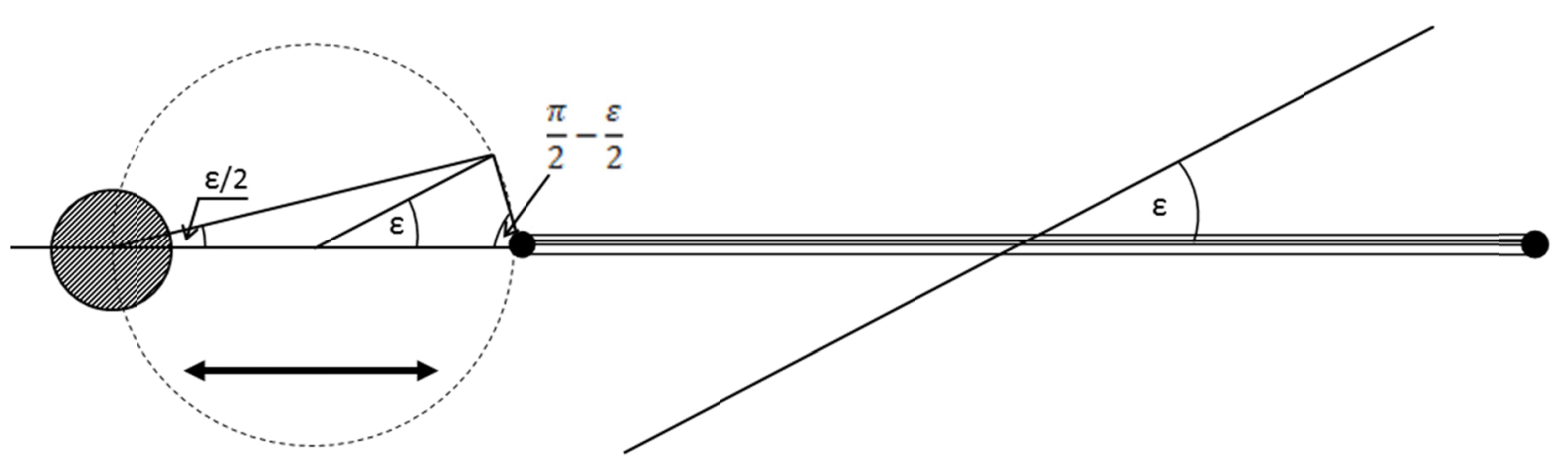

Figure 7. Geometrical configuration of the Karagioz-Izmailov-Gillies-Gershteyn configuration with one source mass

For the description of this gravitational force we will use the trigonometric function "Sagitta" $\sin ^{2}(\varepsilon / 2)$ where $\varepsilon$ is the angle of the horizontal torsion balance towards the constellation Crater. This equation reveals that the Karagioz-Izmailov-Gillies-Gershteyn configuration is dependent on the space orientation towards the constellation Crater:

$$
|F|=\frac{1+\frac{u_{Y}}{c}-2 \frac{u_{Y}}{c} \sin ^{2}\left(\frac{\varepsilon}{2}\right)}{\sqrt{1-\frac{u_{Y}^{2}}{c^{2}}}}\left|F_{0}\right|
$$

Gershteyn et al. (2002) observed a space orientation signal during their seven month long experiments but their experimental approach was not further developed. 


\section{A working Formula for the Newton's Gravitational Law}

There is a tendency to decompose the Newton's gravitational constant into its parts in order to get some additional information about the gravitational field around the source mass.

Our working formula for the decomposition of the Newton's gravitational constant is:

$$
|F|=4 \pi G \frac{M_{S} M_{T}}{4 \pi R^{2}}=\frac{4 \pi R^{2} \lambda}{\sum m_{\text {graviton }}} v \frac{\sum m_{\text {graviton }}}{M_{S} T} \frac{M_{S} M_{T}}{4 \pi R^{2}}
$$

This formula is composed from several parts:

- the first part describes the reciprocal value of the density of gravitons in the spherical shell with radius $\mathrm{R}$ and with the layer thickness $\lambda$ - the wavelength of the gravitational wave,

- the second part is the frequency $v$ of the gravitational wave,

- the third part is the specific mass evaporation constant $\mathrm{H}_{0}$ describing the evaporation of gravitons with mass $\mathrm{m}_{\text {graviton }}$ from the source mass $\mathrm{M}_{\mathrm{S}}$ per unit of time $\left[\mathrm{kg} \mathrm{kg}^{-1} \mathrm{~s}^{-1}\right]$. The numerical value is expected to be identical with the Hubble constant known from the astrophysical experiments,

- $\quad$ the fourth part is the product of the source mass $\mathrm{M}_{\mathrm{S}}$ and the test mass $\mathrm{M}_{\mathrm{T}}$ per spherical surface.

This concept can be experimentally tested:

$$
4 \pi G=\frac{4 \pi R^{2} \lambda}{\sum m_{\text {graviton }}} v \frac{\sum m_{\text {graviton }}}{M_{S} T}=c H_{0} \frac{R^{2}}{M_{S}}
$$

If the source mass $\mathrm{M}_{\mathrm{S}}=1 \mathrm{~kg}$ and the spherical radius equals $\mathrm{R}=1 \mathrm{~m}$ we can get from CODATA $2010 \mathrm{big} \mathrm{G}$ value $=(6.67384 \pm 0.00080)^{*} 10^{-11} \mathrm{~m}^{3} \mathrm{~kg}^{-1} \mathrm{~s}^{-2}$ the product $\mathrm{c}^{*} \mathrm{H}_{0}=8,386 \ldots * 10^{-10} \mathrm{~ms}^{-2}$ and $\mathrm{H}_{0}=86.26 \ldots \mathrm{kms}^{-1} \mathrm{Mpc}^{-1}$. These values depend on the experimental value of the big G. In this case the letter $\mathrm{c}$ describes the longitudinal speed (there is the very well known constant $|\mathrm{c}|$ - the modulus of the light speed - used in the Maxwell equations).

There are three experimental fields that can be used for the test of this concept:

1. Modified Newtonian dynamics MOND - Mordeham Milgrom (1999) introduced a new approach to this field and with many followers continue to develop the MOND concept.

2. Hubble constant experimental search - this is a very active field of research since the time of Edwin Powell Hubble - see the web site of John P. Huchra (2008). The distance of objects for the determination of the Hubble constant plays a very important role. We should try to find this value for objects with very well defined short distances. It will be interesting if we will find the experimental value close to $\mathrm{H}_{0}=86.26 \ldots \mathrm{kms}^{-1} \mathrm{Mpc}^{-1}$ calculated from the big $\mathrm{G}$. See numerous contributions of astrophysicists to this topic.

- Pioneer anomaly: John D. Anderson (1998) discovered the Pioneer anomaly and with co-workers found the anomalous acceleration $\mathrm{a}_{\text {pio }}=(8.74 \pm 1.33)^{*} 10^{-10} \mathrm{~ms}^{-2}$. Though, their interpretation goes into other direction, we should continue in this research and further experimentally tune this acceleration value. It will be interesting to investigate how the values of the big $\mathrm{G}$ and the Hubble constant will behave in further progress with more sophisticated instruments.

\section{Conclusions}

The geometrical configuration of source and test masses for the experimental determination of the Newtonian gravitational constant G plays a very critical role. The old trigonometric function "sagitta" was newly used and a possible trigonometric correction of big $\mathrm{G}$ was proposed. Three geometrical configurations were quantitatively analyzed with predictions: 1) "true" Michell-Cavendish configuration is not dependent on the space orientation - a null experiment. 2) Karagioz-Izmailov configuration with two source masses is expected to be dependent on the space orientation towards the constellation Crater. 3) Karagioz-Izmailov-Gillies-Gershteyn configuration with one source mass is expected to be dependent on the space orientation towards the constellation Crater. One working formula for the decomposition of big $\mathrm{G}$ was presented with proposals for further experimental research. This concept could not be experimentally tested before the discoveries of the dipole in the cosmic microwave background radiation and the Pioneer anomaly. 


\section{Acknowledgments}

This work was supported by the JP\&FŠ Agency (Contract Number 25g/1963), by the VZ\&MŠ Agency (Contract Number 16000/1989) and by the GMS Agency (Contract Number 69110/1992). We have found the valuable support on the web site www.wolframalpha.com with the corrections of used formulae. The valuable support was done by the search engines on the web sites: scholar.google.com and www.google.com. Several important books were delivered by www.amazon.com. Wikipedia was used as an entry gate to numerous original papers.

\section{References}

Anderson, J. D., Laing, P. A., Lau, E. L., Liu, A. S., Nieto, M. M., \& Turyshev, S. G. (1998). Indication, from Pioneer 10/11, Galileo, and Ulysses Data, of an Apparent Anomalous, Weak, Long-Range Acceleration. Physical Review Letters, 81(14), 2858-2861. http://dx.doi.org/10.1103/PhysRevLett.81.2858

Armstrong, T. R., \& Fitzgerald, M. P. (2003). New measurements of G using the measurement standard laboratory torsion balance. Phys. Rev. Lett., 91, 201101. http://dx.doi.org/10.1103/PhysRevLett.91.201101

Baily, F. (1843). An account of some experiments with the torsion-rod, for determining the mean density of the Earth. Memoirs of the Royal Astronomical Society, 14, 1-120. http://dx.doi.org/10.1093/mnras/5.23.188

Beams, J. W. (1964). Method of determining the gravitational constant G. Proc. Virginia J. Sci., 15, 269.

Boys, C. V. (1895). On the Newtonian constant of gravitation. Phil. Trans. R. Soc. London A, 186, 1-72. http://dx.doi.org/10.1098/rsta.1895.0001

Brackenridge, J. B. (1996). The Key to Newton's Dynamics: The Kepler Problem and the Principia. University of California Press, Berkeley. Retrieved from http://ark.cdlib.org/ark:/13030/ft4489n8zn/

Braun, C. (1897). Die Gravitations-Constante, die Masse und mittlere Dichte der Erde nach einer neuen experimentellen Bestimmung. Denkschriften der Akademie von Wissenschaft zu Wien, Mathematische und Naturwissenschaftliche Klasse, 64, 187-258. Retrieved from http://searchworks.stanford.edu/view/7003199

Cavendish, H. (1798). Experiments to determine the density of the Earth. Phil. Trans. R. Soc., 88, 469-526. http://dx.doi.org/10.1098/rstl.1798.0022

Chen, Y. T., \& Cook, A. (1999). Gravitational Experiment in the Laboratory. Cambridge University Press, Cambridge. http://www.fzu.cz/node/95266

CMBR astronomy timeline (2014). In Wikipedia. Retrieved from http://en.wikipedia.org/wiki/Timeline_of_ cosmic_microwave_background_astronomy

Cohen, I.B. (1999). A Guide to Newton's Principia. University of California Press, Berkeley. Retrieved from http://www.ucpress.edu/book.php?isbn=9780520088177

Datta, B., \& Singh, A. N. (1983). Hindu Trigonometry. Indian Journal of History of Science 18, 39-108. Retrieved from http://www.new1.dli.ernet.in/data1/upload/insa/INSA_1/20005af7_39.pdf

De Gandt, F. (1995). Force and Geometry in Newton's Principia. Princeton University Press, Princeton. Retrieved from http://press.princeton.edu/titles/5724.html

Doppler, C. (1842). Über das farbige Licht der Doppelsterne und einiger anderer Gestirne des Himmels. Abhandlungen der Königlichen Böhmischen Gesselschafte der Wissenschaften. V. Folge, Bd., 2, 465-482.

Ducheyne, S. (2011). Testing universal gravitation in the laboratory, or the significance of research on the mean density of the Earth and Big G, 1798-1898: changing pursuits and long-term methodological-experimental continuity. Arch. Hist. Exact Sci., 65, 181-227. http://dx.doi.org/10.1007/s00407-010-0075-9

Einstein, A. (1915), Die Feldgleichungen der Gravitation. Sitzungsberichte der Preussischen Akademie der Wissenschaften zu Berlin. (pp. 844-847). Retrieved from http://articles.adsabs.harvard.edu/cgi-bin/ get_file?pdfs/SPAW./1915/1915SPAW.......844E.pdf

Eötvös, R. (1896). Untersuchungen über Gravitation und Erdmagnetismus. Wiedemanns Annalen, 59, 354-400.

Eötvös, R., Pekár, D., \& Fekete, E. (1922). Beiträge zum Gesetz der Proportionalität von Trägheit und Gravität. Ann. Phys., 68, 11-66. http://www.math.utah.edu/pub/tex/bib/einstein.bib

Galilei, G. (1980). Galileo's notes on motion. Supplemento agli Annali dell'Istituto e museo di storia della scienzia. Retrieved from http://www.museogalileo.it/esplora/biblioteche/bibliotecadigitale.html 
Gershteyn, M. L., Gershteyn, L. I., Gershteyn, A., \& Karagioz, O. V. (2002). Experimental evidence that the gravitational constant varies with orientation. Classical Physics, Gravitation \& Cosmology, 8, 1-6. Retrieved from http://arxiv.org/ftp/physics/papers/0202/0202058.pdf

Gillies, G. (1987). The Newtonian Constant: an index of measurements. Metrologia, 24(Supplement), 1-56. http://dx.doi.org/10.1088/0026-1394/24/S/001

Gillies, G. T. (1997). The Newtonian gravitational constant: recent measurements and related studies. Rep. Prog. Phys., 60, 151-225. http://dx.doi.org/10.1088/0034-4885/60/2/001

Gundlach, J. H., \& Merkowitz, S. M. (2000). Measurement of Newton's constant using a torsion balance with angular acceleration feedback. Phys. Rev. Lett., 85, 2869-2872. http://dx.doi.org/10.1103/PhysRevLett.85. 2869

Heyl, P. R. (1930). A Redetermination of the constant of gravitation. J. Res. Natl. Bur. Std. (U.S.), 5, 1243. Retrieved from http://www.archive.org/details/redeterminationo56124heyl

Hu, Z. K., \& Luo, J. (2004). Progress in determining the gravitational constant with four attractive masses. $J$. Korean Phys. Soc., 45, S128-S131. PACS numbers: 04.80.Cc, 06.20.Jr

Huchra, J. P. (2008). Hubble constant. Retrieved from https:/www.cfa.harvard.edu/ dfabricant/huchra/hubble/

Jolly, P. J. G. (1881). Die Anwendung der Waage auf Probleme der Gravitation. Ann. Phys., 250, 331-355. http://dx.doi.org/10.1002/andp.18812501010

Karagioz, O. V., \& Izmailov, V. P. (1996). Measurement of gravitational constant with a torsion balance. Meas. Tech., 39, 979-987. http://dx.doi.org/10.1007/BF02377461

Karagioz, O. V., \& Izmailov, V. P. (ND). Newtonian Constant of Gravitation. Retrieved from www.wdcb.ru/sep/ GravConst/index.html

Karagioz, O. V., Izmailov, V. P., \& Gillies, G. T. (1999). "Three position" scheme for measurement of the Newtonian gravitational constant. Grav. Cosmol., 5, 155-159. Retrieved from http://rgs.vniims.ru/oldconts.htm

Kleinevoß, U. (2002). Bestimmung der Newtonschen Gravitationskonstante. PhD Thesis, Universität Wuppertal. Retrieved from https://www.deutsche-digitale-bibliothek.de/binary/7SUBNBPTPZBPEZP7T6KN3WYK4IAZH THD/full/1.pdf

Luo, J., \& Hu, Z. K. (2000). Status of measurement of the Newtonian gravitational constant G. Class. Quantum Grav. 17, 2351-2363. http://dx.doi.org/10.1088/0264-9381/17/12/307

Luo, J., Liu, Q., Tu, L. C., Shao, C. G., Liu, L. X., Yang, S. Q., ... \& Zhang, Y. T. (2009). Determination of the Newtonian gravitational constant $\mathrm{G}$ with time-of-swing method. Phys. Rev. Lett., 102, 240801. http://dx.doi.org/10.1103/PhysRevLett.102.240801

Luther, G. G., \& Towler, W. R. (1982). Redetermination of the Newtonian gravitational constant G. Phys. Rev. Lett., 48, 121-123. http://dx.doi.org/10.1103/PhysRevLett.48.121

Maor, E. (1998). Trigonometric delights. Princeton University Press, Princeton. Retrieved from http://ip144.qb.fcen.uba.ar/libroslfp/Algebra\%20\&\%20Trigonometria/Maor\%20-\%20Trigonometric\%20De lights\%20\%28Princeton,\%201998\%29.pdf

Matvievskaya, G. P. (1990). An outline of the history of science. Tashkent, Fan.

Matzka, W. (1850). Versuch einer richtigen Lehre von der Realität der vorgeblich imaginären Grössen der Algebra, oder einer Grundlehre von der Ablenkung algebraischer Grössenbeziehungen. Prague: J.G.Calve.

Michaelis, W., Haars, H., \& Augustin, R. (1995). A new determination of Newton's gravitational constant. Metrologia, 32, 267-276. http://dx.doi.org/10.1088/0026-1394/32/4/4

Milgrom, M. (1999). The Modified Dynamics as a vacuum effect. Physics Letters A, 253(5-6), 273. http://dx.doi.org/10.1016/S0375-9601(99)00077-8

Milyukov, V. K., \& Fan, S. H. (2012). The Newtonian Gravitational Constant: Modern Status of Measurement and the New CODATA Value. Gravitation and Cosmology, 18, 216-224. http://dx.doi.org/10.1134/S0202289312 030061

Milyukov, V. K., Luo, J., Tao, C., \& Mironov, A. P. (2008). Status of the experiments on measurement of the Newtonian gravitational constant. Gravitation and Cosmology, 14(4), 368-375. http://dx.doi.org/10.1134/ S0202289308040130 
Mohr, P. J., Taylor, B. N., \& Newell, D. B. (2012). CODATA recommended values of the fundamental physical constants: 2010. Rev. Mod. Phys., 84, 1527-1605. http://dx.doi.org/10.1103/RevModPhys.84.1527

Newton, I. (1687). The Principia. Mathematical of Principles of Natural Philosophy. In I. B. Cohen, \& A. Whitman (Translated). Berkeley: University of California Press. Retrieved from http://philpapers.org/sep/ newton-principia/

Panjwani, H. (2012). Development of a torsion balance facility and a search for temporal variations in the Newtonian gravitational constant. PhD Thesis, University Birmingham.

Philosophical Transaction A. (2014). "Theo Murphy Meeting Issue 'The Newtonian constant of gravitation, a constant too difficult to measure?"' Organized and edited by Terry Quinn and Clive Speake (Vol. 372, issue 2026). http://dx.doi.org/10.1098/rsta.2014.0286

Planck mission. (2013). In NASA, Jet Propulsion Laboratory. Retrieved from http://planck.caltech.edu/ publications2013Results.html

Poynting, J. H. (1894). The Mean Density of the Earth. Charles Griffin Company, London. Retrieved from www.forgottenbooks.com/

Quinn, T. J., Parks, H., Speake, C. C., \& Davis, R. S. (2013). Improved determination of G using two methods. Phys. Rev. Lett., 111, 101102. http://dx.doi.org/10.1103/PhysRevLett.111.101102

Quinn, T. J., Speake, C. C., Richmann, S. J., Davis, R. S., \& Picard, A. (2001). A new determination of G using two methods. Phys. Rev. Lett., 87, 111101. http://dx.doi.org/10.1103/PhysRevLett.87.111101

Reich, F. (1852). Neue Versuche mit der Drehwaage. Abhandlungen der mathematisch-physikalischen Classe Königlichen Sächsischen Gesselschaft der Wissenschaft., 1, 384-430. Retrieved from $\mathrm{http} / /$ www.worldcat.org/title/neue-versuche-mit-der-drehwaage/oclc/18535410?referer=di\&ht=edition

Richarz, F., \& Krigar-Menzel, O. (1898). Bestimmung der Gravitationsconstante und mittleren Dichtigkeit der Erde durch Wägungen. Anhang zu den Abhandlungen der Königlichen Preussischen Akademie der Wissenschaften zu Berlin, 1-196. Retrieved from http://jfm.sub.uni-goettingen.de/cgi-bin/jfmen/JFM/en/ quick.html? first $=1 \&$ maxdocs $=20 \&$ type $=$ html\&an $=J F M \% 2027.0665 .01 \&$ format $=$ complete

Rose, R. D., Parker, H. M., Lowry, R. A., Kulthau, A. R., \& Beams, J. W. (1969). Determination of the gravitational constant G. Phys. Rev.Lett., 23, 655-658. http://dx.doi.org/10.1103/PhysRevLett.23.655

Sagitov, M. U. (1970). Current status of determinations of the gravitational constant and the mass of the Earth. Soviet Astronomy, 13, 712-718. Retrieved from http://articles.adsabs.harvard.edu

Schlamminger, S. (2002). Determination of the gravitational constant using a beam balance. PhD Thesis, Universität Zurich. http://www.schlammi.com/pdf/diss.pdf

Schumacher, A. (1999). Systematische Untersuchungen zur Messung der Newtonschen Gravitationskonstante mit einem Pendelresonator. PhD. Thesis, Universität Wuppertal. Retrieved from http://www.bib. uni-wuppertal.de/elpub/fb08/diss1999/schumacher/d089907.pdf

Schwartz, J. P. (1998). The free-fall determination of the universal constant of gravity. PhD Thesis, University of Colorado. Retrieved from http://jila01.colorado.edu

Speake, C. C. (2005). Newton's constant and the 21 st century laboratory. Phil. Trans. R. Soc. A, 363, 2265-2287. http://dx.doi.org/10.1098/rsta.2005.1643

Speake, C. C., \& Quinn, T. S. (1999). Special edition: The gravitational constant: theory and experiment 200 years after Cavendish. Meas. Sci. Technol., 10, 422-530. http://dx.doi.org/10.1088/0957-0233/10/6/001

Stávek, J. (2013). On the one trigonometric interpretation - the decomposition loophole. App. Phys. Res., 5(6), 131-144. http://dx.doi.org/10.5539/apr.v5n6p131

Stávek, J. (2013). On the trigonometric loophole. Appl. Phys. Res., 5(6), 48-58. http://dx.doi.org/10.5539/apr.v5n6p48

Unnikrishnan, C. S., \& Gillies, G. T. (2002). Nano-constraints on the spatial anisotropy of the gravitational constant. http://dx.doi.org/10.1016/S0375-9601(02)01390-7

Van Brummelen, G. (2009). The mathematics of the Heavens and the Earth (The early history of trigonometry). Princeton: Princeton University Press. Retrieved from http://press.princeton.edu/titles/8956.html 
Van Brummelen, G. (2013). Heavenly mathematics (The forgotten art of spherical trigonometry). Princeton: Princeton University Press. Retrieved from http://press.princeton.edu/titles/9834.html

Voigt, W. (1887). Über das Doppler'sche Prinzip. Göttinger Nachrichten, 2, 41-51. Retrieved from http://en.wikisource.org/wiki/Translation:On_the_Principle_of_Doppler

Von Braunmühl, A. (1900). Vorlesungen über Geschichte der Trigonometrie. Leipzig, Teubner. Retrieved from http://catalog.hathitrust.org/Record/000165837

Whiteside, D.T., Hoskin, M.A. \& Prag, A. Geometry and the Dynamics of Motion. In The Mathematical Papers of Isaac Newton, VI, 1684-1691, Cambridge University Press, Cambridge. http://dx.doi.org/10.1017/S0007087 400014576

Wilsing, J. (1887). Bestimmung der mittleren Dichtigkeit der Erde mit Hilfe eines Pendelapparates. Publikationen des Astro-physikalischen Observatoriums zu Potsdam, 22, 35-127. Retrieved from http://d-nb.info/560188692

Xue, C., Quan, L. D., Yang, S. Q., Wang, B. P., Wu, J. F., Shao, C. G., ... \& Luo, J. (2014). Preliminary determination of Newtonian gravitational constant with angular acceleration feedback method. Philosophical Transactions of the Royal Society A: Mathematical, Physical and Engineering Sciences, 372(2026), 20140031. http://dx.doi.org/10.1098/rsta.2014.0031

Zahradníček, J. (1933). Resonanzmethode für die Messung der Gravitationskonstante. Physik. Zeitschr., 34, 126-133.

\section{Copyrights}

Copyright for this article is retained by the author(s), with first publication rights granted to the journal.

This is an open-access article distributed under the terms and conditions of the Creative Commons Attribution license (http://creativecommons.org/licenses/by/3.0/). 